\title{
Understanding How Work Habits influence Student Performance
}

\author{
Seth Copen Goldstein \\ ${ }^{*}$ Carnegie Mellon University \\ Pittsburgh, PA \\ seth@cmu.edu
}

\author{
Hongyi Zhang \\ Peking University \\ Beijing, China \\ hongyizhang@pku.edu.cn
}

\author{
Majd Sakr* \\ msakr@cs.cmu.edu
}

\author{
Haokang $\mathrm{An}^{*}$ \\ haokanga@andrew.cmu.edu
}

\begin{abstract}
Understanding the relationship between a student's broader work habits and their performance, particularly on open-ended programming assignments, is key towards being able to guide students towards success. In spite of this, most evidence of student behavior and its relationship to performance is anecdotal. The advent of large-scale courses which use online tools for delivering course content, monitoring the programming environment, and providing automatic feedback as well as grading now makes it possible to dive into the data and develop data-driven methods for understanding how a student's approach to an assignment-from their first exposure to the description of the problem to their final submission of their completed assignment-influences their final performance.

This study is a first look at a subset of the data collected from a project-based, online, upper-level course on cloud computing. We examine three raw data streams which include information on the time students spend on reading the project write-up, the timing, grades, and number of submissions they make, and the cloud resources used (both time and cost) in solving the assignment. Using these and several synthetic metrics we were surprised to find that there are few behaviors that are highly correlated to student success. Instead, we find that there is a strong correlation between students who continually apply consistent behaviors over the course of the semester to good final performance in the course. From this we use LASSO to create a predictor (adjusted $R^{2}=.48$ ) of final performance based on two kinds of consistency measures across 15 metrics.
\end{abstract}

\section{KEYWORDS}

Online education; student performance; educational data mining; learning analytics; project-based learning; work habits

\section{ACM Reference Format:}

Seth Copen Goldstein, Hongyi Zhang, Majd Sakr, Haokang An, and Cameron Dashti. 2019. Understanding How Work Habits influence Student Performance. In Innovation and Technology in Computer Science Education (ITiCSE '19), July 15-17, 2019, Aberdeen, Scotland Uk. ACM, New York, NY, USA, 7 pages. https://doi.org/10.1145/3304221.3319757

Permission to make digital or hard copies of all or part of this work for personal or classroom use is granted without fee provided that copies are not made or distributed for profit or commercial advantage and that copies bear this notice and the full citation on the first page. Copyrights for components of this work owned by others than ACM must be honored. Abstracting with credit is permitted. To copy otherwise, or republish, to post on servers or to redistribute to lists, requires prior specific permission and/or a fee. Request permissions from permissions@acm.org.

ITiCSE '19, fuly 15-17, 2019, Aberdeen, Scotland Uk

(c) 2019 Association for Computing Machinery.

ACM ISBN 978-1-4503-6301-3/19/07 . \$ \$15.00

https://doi.org/10.1145/3304221.3319757

\section{INTRODUCTION}

There is a significant literature on how students work and the impact it has on their performance; sometimes even on how they learn. Until recently most of these studies were limited to small populations and/or survey based approaches because students did most of their work in an unobservable way. While there are numerous studies, they often come to conflicting conclusions, e.g., more effort is either positively or negatively correlated with performance. With the advent of online courses, more data can be collected. However, even studies based on this new source of data yield conflicting results. All of these conflicting studies exist against a backdrop of teachers making well meaning suggestions to students to help them do well: start your project early, read the entire write-up before doing any coding, take lots of short breaks, spread your work over a few days, don't pull all-nighters, etc. Unfortunately, there is no real data to suggest these suggestions are useful.

This study is a first look at data that covers how students work on projects from the moment they get the write-up on a Learning Management System (LMS) to the time of their final submission on an auto grading system. We collect 15 data streams from 11 different one-week projects over the course of a single semester of an online upper-division project-based course.

While our ultimate goal is to identify which work habits are correlated with the most learning; we instead use grades as a proxy for learning and look at which work habits are correlated with the best performance. We had hoped to identify which habits are best; to support (or refute) the common wisdom often given to students. However, we soon found that few habits are good for all students. As we discuss in Section 3, we find that there is little evidence that any one work habit is best for all students. In retrospect, this is not surprising as it is well known that different people have different work styles $[4,8]$. Further investigation suggests that there is a significant and strong positive correlation between the consistency in how they tackle each project and their final performance.

We describe our infrastructure and how we collect the data in Section 2. We present our main results which lead to a useful predictor of performance which explains more than $40 \%$ of the variance in our data in Section 3. We briefly outline related work in Section 4 and conclude with some ideas for future work in Section 5.

\section{METHODOLOGY AND DATA COLLECTION}

We investigate whether specific student behavior leads to improved performance in a project-based upper-level CS course. We attempt to find a correlation between direct and synthetic student behavior metrics (see Table 1) and performance. We analyze whether the temporal consistency of student behavior and performance are correlated. Finally, we propose a model for performance prediction. 


\begin{tabular}{|c|c|c|}
\hline Metric & Description & Data Sources \\
\hline Starting Early & The negative of the number of hours into the week they start seriously reading the write-up. & pLMS \\
\hline Finishing Early & The number of hours before the project deadline that they make their the last submission. & AGS \\
\hline Write-up Completion & The percentage of the handout that was read before the first submission was made. & pLMS + AGS \\
\hline Wall-Clock Time & $\begin{array}{l}\text { Total wall-clock time between when they first start reading the write-up and when they } \\
\text { make their final submission. }\end{array}$ & pLMS + AGS \\
\hline Short Breaks & $\begin{array}{l}\text { The number of short breaks a student takes. A break is a period of less than } 1 \text { hour of no } \\
\text { activity bordering work activity of at least } 1 \text { hour. }\end{array}$ & pLMS, AGS, CSP \\
\hline Work Periods & $\begin{array}{l}\text { The number of periods of contiguous activity broken up by periods of inactivity of more } \\
\text { than } 5 \text { hours. }\end{array}$ & pLMS, AGS, CSP \\
\hline Total Active Time & $\begin{array}{l}\text { The total amount of time the student is working on the project; derived from reading the } \\
\text { write-up, using cloud resources, and making submissions. }\end{array}$ & pLMS, AGS, CSP \\
\hline Pre-submission Time & $\begin{array}{l}\text { The time between when the student starts seriously reading the write-up and their first } \\
\text { submission. We measure this both for wall-clock time and active time. }\end{array}$ & pLMS + AGS \\
\hline Number of Submissions & The total number of submissions made to the autograder, both per project and per task. & AGS \\
\hline ECR Cycle Time & Median time between submissions of the same task. & AGS \\
\hline Submission Grade Delta & The median difference in the scores between two submissions. & AGS \\
\hline Cloud time & The total time that cloud resources are instantiated for the project. & CSP \\
\hline Cost & The percentage of the allocated budget that the student uses to complete the project. & CSP \\
\hline
\end{tabular}
Table 1: Metrics, how we calculate them, and what their source is.

\begin{tabular}{r|c} 
Metric & Correlation \\
\hline Starting Early & $0.198 *$ \\
Finishing Early & $0.444 * * *$ \\
Write-up Completion & -0.130 \\
Wall-Clock Time & -0.043 \\
Short Breaks & 0.093 \\
Work Periods & 0.124 \\
Total Active Time & 0.105 \\
Pre-submission Activity Time & $-0.279 * * *$ \\
Pre-submission Wall-clock Time & $-0.385 * * *$ \\
Number of Submissions & $0.221 * *$ \\
Number of Submissions/Task & 0.074 \\
ECR Cycle Time & -0.135 \\
Submission Grade Delta & -0.004 \\
Cloud Time & -0.097 \\
Cost & 0.013
\end{tabular}

Table 2: Correlation between metrics as described in Table 1 and final performance.

The data was collected from a semester-long online projectbased course offered to students at Carnegie Mellon University's four global campuses in Spring 2018. The course, cross-listed as an undergraduate and graduate course, requires students to complete 11 individual projects and (for the graduate students) to participate in a team project. Since this is an online course, there are no lectures to attend. Students in this online course complete conceptual topics and assessments on an LMS platform and use another homegrown platform, TheProject.Zone, to access and submit solutions to the programming projects. Students in the course interact with the teaching staff and with other students on Piazza, a question and answer forum. The course has been offered 10 times before in the same format. The data collected and reported on in this paper are from undergraduate and graduate students working on the individual projects only.

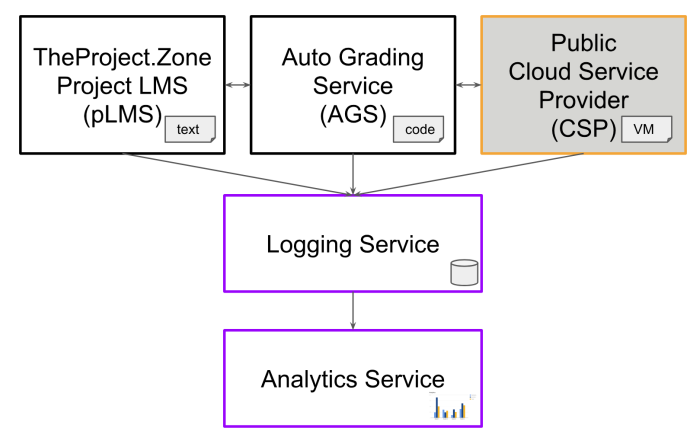

Figure 1: We use three data sources to infer behavior, logs from: the pLMS where students access the project write-up, the AGS where students submit their solutions and get automated and contextualized feedback, and from the CSP which indicate when students provision cloud resources as well as their cost.

Data were collected from 154 students enrolled in our course in the Spring 2018 semester. The data sources (see Fig. 1) include TheProject.Zone, a project LMS (pLMS), the Auto Grading Service (AGS) and student logs from provisioning resources on a public Cloud Service Provider (CSP). From the pLMS, we collected their heartbeat time and their scoreboard data. Heartbeat is an indicator that students are currently interacting with the project write-up. It is recorded every 5 minutes to capture student online behavior continuously, and we can trace down to the specific write-up section the student is reading. Scoreboard data presents students' score for each task and each project, and it is visible to both the instructor and the students. From AGS, we collected their submission information and the corresponding feedback, from which we can see how the student score increases over time as they go through the whole project. From CSP, we collected their cloud resource running time and the corresponding cost. Every project is associated with a particular budget, and the students should monitor and manage their cloud resources so that they do not go over the budget or their score is reduced. 


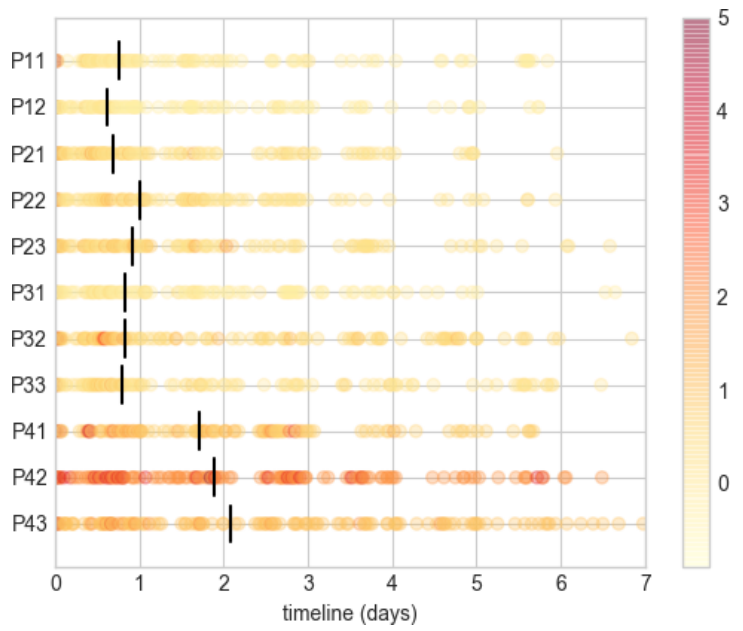

Figure 2: The time students start reading the project write-up. (Vertical bars in this and future figures indicate the median value.)

While most of the metrics we discuss come directly from the data sources described here, we do have to estimate the time that students are actually working on the project. We call it the Total Active Time in Table 1, to account for the time that the students are working offline or on their local computer. We use the heartbeat from the pLMS as the basis for their active time. Submission activity from the AGS is included as an hour worth of work if there is no heartbeat within an hour of the submission, or we consider the student as working during the interval between the closest heartbeat and the time of submission. Finally, we include from the CSP the time that cloud resources are running as long as those resources are running for less than 12 hours. (If they are running for more than that, we assume the student forgot to turn them off.) The total active time is the union of the heartbeat time, the merged submissions, and the cloud resource running periods.

While we cannot capture all student behavior-i.e., some of their work may happen off-line when they are not engaged with the data sources-we are capturing most of it as indicated by comparing the self-reported time spent on each project (from post-project survey data) and the estimated active time. Of the eleven individual projects, three projects have students reporting (in aggregate) that they spend between $1.2 \mathrm{x}$ and $1.7 \mathrm{x}$ our estimate while all the projects have student reported times which are $\pm 10 \%$ of our estimate.

\section{EXPERIMENTS}

The ultimate goal of this research is to understand how students learn and then to develop interventions which aid their learning. Here we present a preliminary evaluation of some of the data we have collected with the caveat that measuring learning, particularly in the case of a single course, is difficult at best. Thus, we look at how student behavior relates to final performance (i.e., grades) as a poor, but measurable, proxy for learning. We hope that understanding how student behavior is related to student performance will shed some light on how students succeed and indirectly lead us to understand the best way to enable student learning in future work. Table 1 describes the data sources that we examine, how we gather them, and when appropriate how we calculate them.

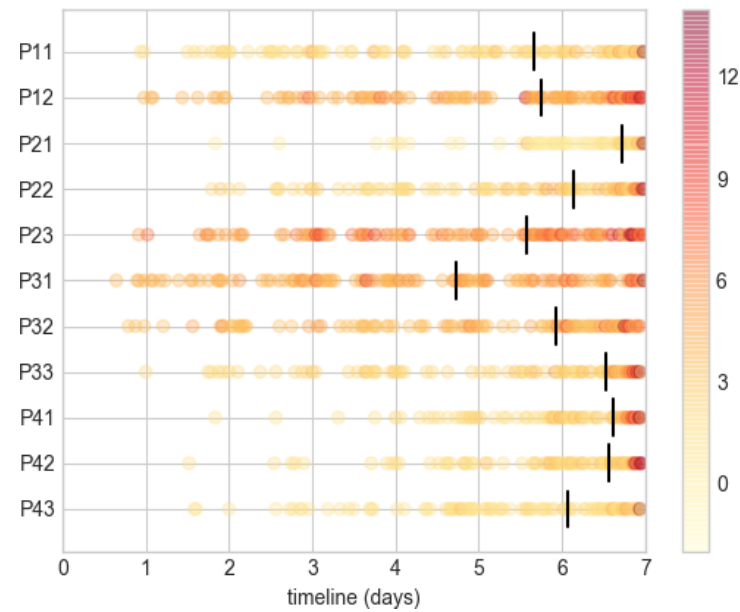

Figure 3: The time at which students finish the project.

\subsection{Direct Work Habits}

Here we look at different work habits and their relationship to performance. The correlation and significance between each metric and the final performance of the student can be found in Table 2.

One work style, often praised by faculty, is starting a project early. Because the write-up for each project is delivered online we can measure when students begin the project by recording when a student begins seriously reading ${ }^{1}$ the write-up. As Fig. 2 shows, students generally start reading the project quite early in the week, but starting times can vary quite a bit. Surprisingly, the correlation between starting early and doing well on a project is only slightly significant. (See the first row, labeled Start Early, of Table 2.)

We do, however, find a strong and significant correlation $(r=$ $\left.0.444^{* * *}\right)^{2}$ between how early a student finishes the project (measured in how many hours before the deadline they make their final submission) and their performance on the project. (See row two, labeled Finishing Early, Table 2.) Fig. 3 shows the distribution of end times for our 11 projects. Interestingly, the correlation is not strictly an artifact of students stopping when they get a perfect score. Depending on the project, less than half the students who finish early have a perfect score. While we cannot be sure what work habit this is linked to, our hypothesis is that busy students who are good at managing their time are likely to realize that it is not worth the extra time to get only a marginally better score. Alternatively, finishing early may be a sign that the students are not procrastinating; which is significantly correlated with performance $[3,6,12]$.

Generally, we advise students to read the entire write-up before beginning work on the project. The percentage of the write-up read by students before they make their first submission varies significantly. Contrary to our intuition, there is no evidence that this impacts performance either positively or negatively. (See row labeled Write-up Completion of Table 2.)

The next four rows of Table 2 deal with how students allocate their time on a project. Wall-Clock Time refers to the total amount of time between when they first start reading the write-up until

\footnotetext{
${ }^{1}$ By "seriously reading," we mean more than just glancing at the write-up. I.e., they must be interacting with the write-up for more than 5 minutes to count as "seriously reading" it.

${ }^{2}$ Whenever we include correlation numbers we also include their significance level: * $p<.05 ;{ }^{* *} p<.01 ;{ }^{* * *} p<.001$.
} 


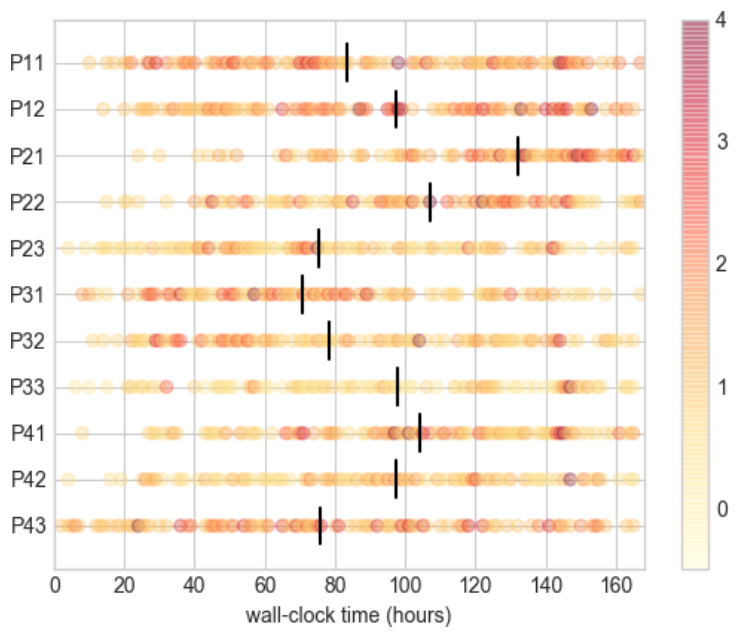

Figure 4: The wall clock time from the start to the end of the project.

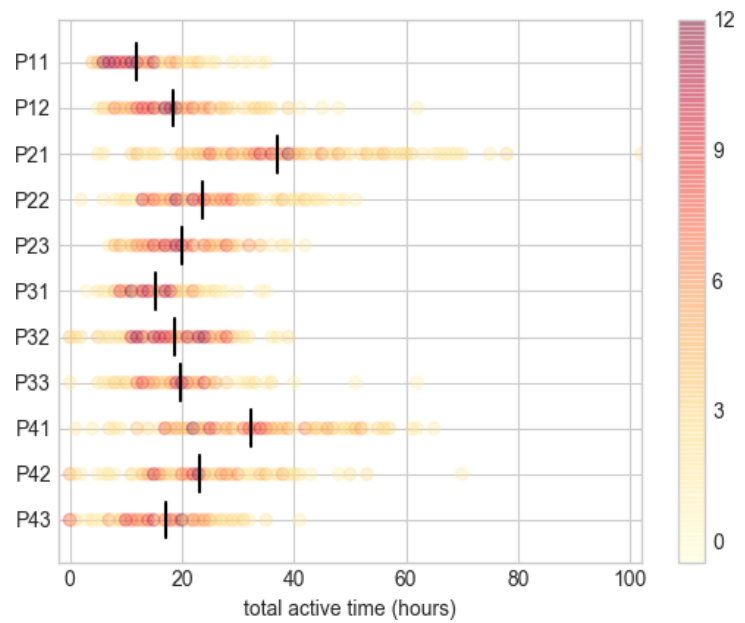

Figure 5: The total amount of time (derived) that students spend on a project.

their final submission. In other words, do they spread out their work over the week (a long wall-clock time), or strongly focus on this project alone (a shorter start to end period). As Fig. 4 shows, students vary significantly in how they focus on the project. Short Breaks is an indication of how many short breaks the student takes during the course of working on the project. And, Work Periods (see different distribution of work periods in Fig. 7.) refers to how many long sessions the student takes to complete the project. Contrary to standard intuition none of these work habits significantly correlate with final performance. It is no surprise then, that the Total Active Time spent on the project (which varies significantly as shown in Fig. 5.) is also not correlated with final performance.

We can determine not only how long students work, but also when they work. The day-night cycles are clearly evident in Fig. 6 , as is the fact that students tend to pull an all-nighter for the harder projects, e.g., P21 and P33. Other artifacts from the course are also evident, E.g., P41, P42, and P43 run coincidentally with the team project and many students seem to focus on the team project, delaying work on the individual projects to the end of the week.

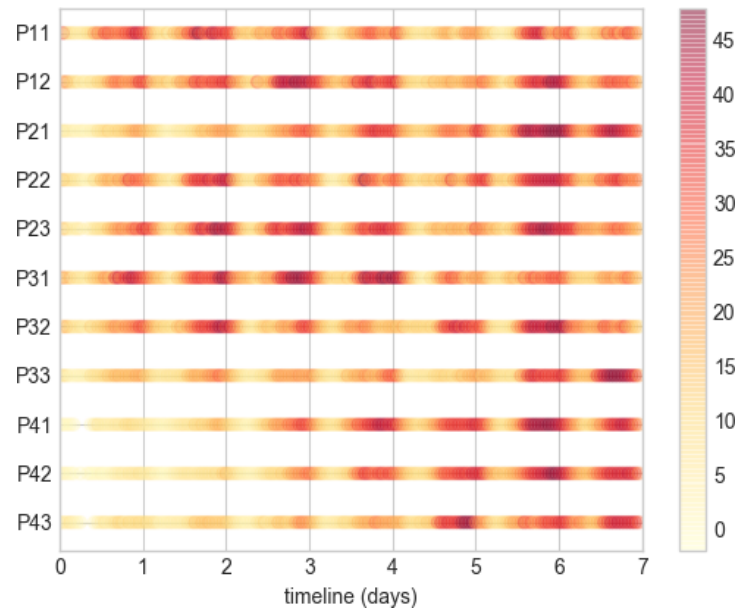

Figure 6: A depiction of when students work on each project.

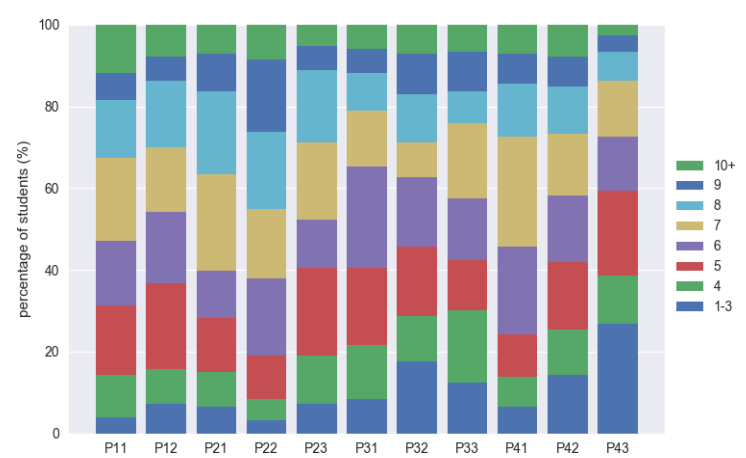

Figure 7: Number of periods students take to finish a project.

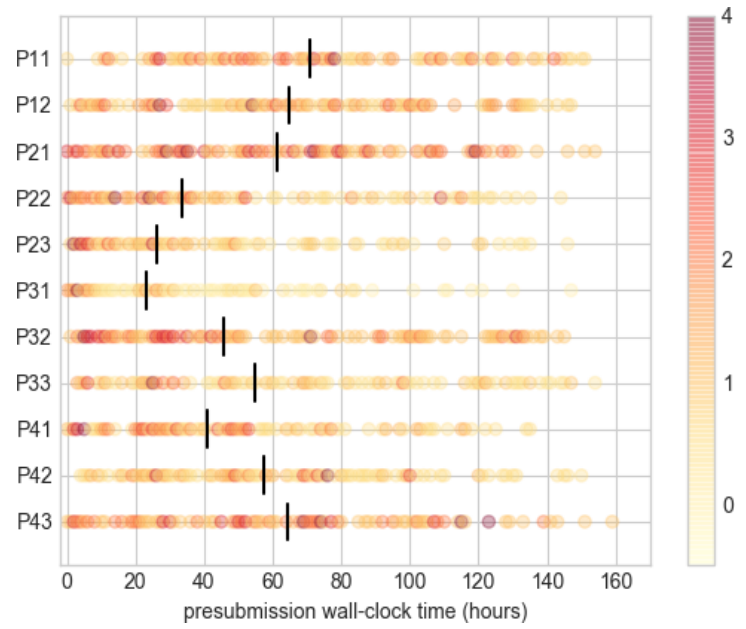

Figure 8: The wall-clock time a student spends working on the project before their first submission.

The aforementioned metrics are easy to collect and directly reflect a work habit. We now look at metrics collected from the AGS, the times and scores of project submissions, from which we infer student behaviors. Pre-submission Time is the time students spend before submitting their first solution. We look at both the wall-clock time (See Fig. 8) and active time (See Fig. 9). Since we do not have data that reflects when they actually start coding, we use this as 


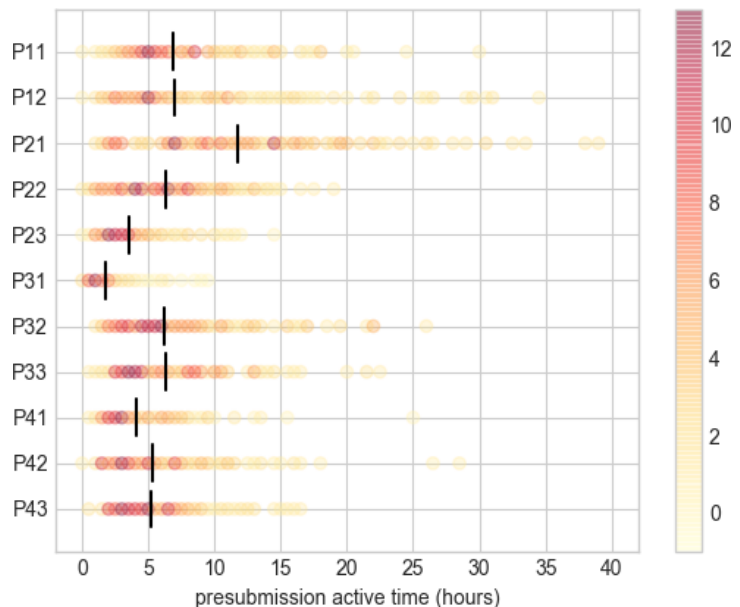

Figure 9: The active time a student spends working on the project before their first submission.

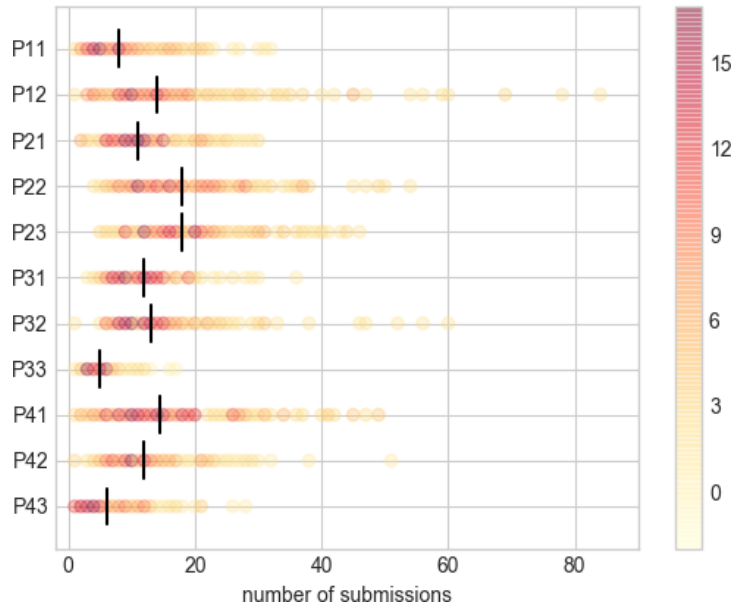

Figure 10: Number of submissions made per student per project.

a proxy for how much time (and thought) the students are giving to the project before they dive into coding. Surprisingly, there is a strong and significant negative correlation between how much time is spent before they make their first submissions $\left(r=-0.385^{* * *}\right.$ for wall-clock time and $r=-0.279^{* * *}$ for active time). Possibly this metric is not a proxy for thinking time, but a proxy for procrastination; possibly supported by the fact that wall-clock time is more negatively correlated with their final performance than active time.

Students almost always need to make more than one submission to reach their final grade. The time between submissions and the change in scores of these submissions could be proxies for how the students approach development and debugging. Are they thinking hard between each edit-compile-run (ECR) cycle? Are they practicing test-driven development? Or, are they using the AGS as a debugging tool? Except for the total number of submissions, for which we find a moderately significant positive correlation $(r=$ $\left.0.221^{* *}\right)$, none of these behaviors have any significant relationship to final performance. The strong correlation between the number of submissions and final performance is a perfect example of how hard it is to untangle performance and learning. One possible interpretation of students who submit often is that they are using

\begin{tabular}{r|cc} 
Metric & Absolute & Relative (By Rank) \\
\hline Starting Early & 0.004 & $0.290 * * *$ \\
Finishing Early & $0.514 * * *$ & $0.235 * *$ \\
Write-up Completion & 0.009 & 0.047 \\
Wall-Clock Time & $0.162 *$ & $0.222 * *$ \\
Short Breaks & $0.188 *$ & $0.252 * *$ \\
Work Periods & $0.216 * *$ & 0.077 \\
Total Active Time & $0.235 * *$ & $0.374 * * *$ \\
Pre-submission Activity Time & 0.011 & -0.005 \\
Pre-submission Wall-clock Time & $-0.161 *$ & $0.227 * *$ \\
Number of Submissions & $0.454 * * *$ & $0.337 * * *$ \\
Number of Submissions/Task & $0.249 * *$ & $0.337 * * *$ \\
ECR Cycle Time & -0.048 & -0.012 \\
Submission Grade Delta & 0.015 & 0.093 \\
Cloud Time & $0.292 * *$ & $0.332 * * *$ \\
Cost & $0.480 * * *$ & $0.390 * * *$ \\
Significance levels: ${ }^{*} p<.05 ;{ }^{* *} p<.01 ;{ }^{* * *} p<.001$
\end{tabular}

Table 3: Correlation between the consistency of each metric (as described in Table 1) over all projects and final performance.

the AGS as a debugging tool-something that is probably hindering their learning, but is an effective way to get a good grade. In the future, we hope to design a submission mechanism that will allow us to determine if this is true and encourage students to engage more deeply in the debugging of their code.

The last two metrics we analyze are specific to the cloud computing course from which we collected this data. Each project requires that the students use cloud resources and that they do so with a fixed dollar budget. We expect that the total amount of time that they have resources spun up (the row labeled Cloud Time) to reflect the students' intentionality; less time being related to more intentionality. Likewise, since they have a budget requirement (labeled Cost in Table 2), the less they spend to complete the project the more likely they are to have exhibited intentionality in their work habits. Notice how both of these metrics have a correlation of close to zero and furthermore are insignificant.

Overall we find that the amount of time they spend (on the clock or actively working) and how early they finish to be the only significant metrics with respect to their final performance. (This remains true on a per-project basis as well, though we don't present the data here for lack of space.) At first glance this is surprising, but on reflection, we think this is reasonable-no one work habit or work style is best for everyone, so no one work habit is likely to be significantly correlated with good performance.

\subsection{Consistent Work Habits}

In pursuit of our goal of understanding how to help students improve their learning and overall performance we hoped to discover some underlying "truths," i.e., habits that promote success. The data, however, suggests that there is no one size fits all approach. This begs the question: are there any habits that are useful for individual students? To explore this we looked to see if students who consistently applied the same behaviors over the course of the semester performed well. We used two different consistency measures. We measured absolute consistency following [15] as the negative of the coefficient of variation $(\mathrm{CoV})$ over the metric (to compare on the same scale) over the course of the semester, i.e., 
the ratio of the negative of the standard deviation $\sigma$ to the mean $\mu^{3}$. This is in column two of Table 3 . We also look at the relative consistency, defined as the standard deviation of the percentile rank for each metric of each student among the entire class. See the last column of Table 3 . We developed the relative consistency measure because it is invariant under linear transformations and thus more robust than the absolute $\mathrm{CoV}$ measure. For example, depending on whether we use " $x$ hours before the deadline" or " $7 \times 24-x$ hours into the project" as the finish time will dramatically change the $\mathrm{CoV}$ metric from $\sigma / \mu$ to $\sigma /(7 \times 24-\mu)$, but leaves relative consistency unchanged.

By looking at Table 3 we can immediately see that students who perform consistently during the semester do better than those who do not. The $\mathrm{CoV}$ measure has seven highly significant and positive correlations and the relative measure has 10 positive and significant correlations. In other words, students who have found a habit that works for them and stick to it do well. This does not mean that the student was consistently using the "best" method, but rather that they were using a method that worked for them. So, students who consistently spent almost the entire budget along with students who were consistently parsimonious both performed well.

The three most important metrics, in terms of consistent behavior, are being consistent about finishing at the same time each week, using the same method of development (as measured by consistency of submission count), and about spending the same proportion of their budget. The only metrics with negative correlations (which would suggest that being consistent is harmful) are not significant.

\begin{tabular}{rc} 
Variables & adjusted $R^{2}$ \\
\hline Direct metrics (from Table 2) & 0.260 \\
Absolute Consistency (column 2 of Table 3) & 0.459 \\
Relative Consistency (column 3 of Table 3) & 0.310 \\
Absolute + Relative Consistency & 0.483
\end{tabular}

Table 4: Adjusted $R^{2}$ of different predictors created with LASSO using 5 -fold cross validation.

\subsection{Predicting Performance}

The information presented sheds light on student behavior and begins to explain how it influences their final performance. To test out whether this is useful in predicting importance we develop a prediction model using LASSO to perform model selection with $k$ fold cross validation $(k=5)^{4}$ to determine the best-fit model. Table 4 shows the different predictors and their adjusted $R^{2}$. We see that the consistency measures produce a better predictor than the direct measures. The best predictor is produced when we use both the absolute and relative measures together, yielding a predictor which explains more than $40 \%$ of the variance in our data.

\section{RELATED WORK}

Several studies investigate how student behavior, in particular time spent, is related to their final performance. Some studies [2, 11, 20]

\footnotetext{
${ }^{3}$ We flip the sign on $\mathrm{CoV}$ so our measure is more intuitive, i.e., more positive numbers indicate more consistency

${ }^{4}$ We partition the dataset into five equal-sized sub-samples and use one sample for validation and the rest for training. We perform LASSO five times, using a different sub-sample for validation each time, The five results are then averaged to evaluate the model and choose the best regularization parameter.
}

report, as one would intuitively expect, a positive correlation between time spent and final performance. Yet others [16, 18, 21], suggest that effort is a weak predictor of student performance. Finally, [10], finds that the relationship between effort and performance is complicated and depends on the course considered.

Few studies consider the longitudinal aspect of individual students and the issue of dynamic change in student behavior remains under-explored. Some studies $[1,19]$ found that the most visited part of the course website viewed by all students can change over time, and gradual transitions from one part to another can be observed. From the perspective of individual students, Kinnebrew et al. [7] focused on the delta of certain behavior patterns over time. Other studies investigated the stability of students' learning profile for those enrolled twice in the same course [5, 9]. All these findings, however, have not related temporal evolution to student performance. Nevertheless, some studies [14] take performance into account, and suggest increased student activity may lead to better performance.

Unfortunately, there is no standard definition of "consistency." Studies in face-to-face course settings [17] suggest that consistent attendance leads to better performance. Employing a quantitative approach, Patron and Lopez [15] defined behavior consistency as the coefficient of variation of the amount of time spent on the course each day, and suggested that consistency is more predictive of the final performance than the effort students put into the course. Alternatively, Mojarad et al. [13], proposed consistency to be the average time between two actions, and showed students with higher consistency (in their sense) and effort tend to have better performance. It has also been pointed out that inconsistency may be due to students' procrastination or laziness [22]. This is partly verified by the negative correlation between the level of procrastination and performance for online students as reported in $[3,6,12]$.

\section{CONCLUSIONS}

This study investigates whether student behavior or the temporal consistency of behavior can predict performance in a project-based, online, upper-level course on cloud computing. Using direct and synthetic metrics we find that few behaviors are highly correlated to student performance, but that there is a strong and significant correlation between temporally consistent behaviors over the course of the semester and final performance. This strongly suggests that when analyzing learning data or designing interventions, personal habits and behaviors must be taken into account. Using our findings we create a good predictor (adjusted $R^{2}=.48$ ) of final performance based on two measures of student consistency across 15 metrics.

We hope to expand our study by increasing the granularity for certain metrics, e.g., the pLMS heartbeat, and incorporating other data sources, e.g., when and what questions students ask. With the improved data we hope to identify behavior inconsistency earlier in the semester and trigger possible interventions. Furthermore, we plan to include an assessment of pre- and post-project skills so we can more directly tie our study to actual learning.

\section{ACKNOWLEDGMENTS}

This work is supported in part by an AWS Educate grant, Microsoft Azure Educator Grant Award and a Google Cloud Platform grant. 


\section{REFERENCES}

[1] Hercy NH Cheng, Zhi Liu, Jianwen Sun, Sanya Liu, and Zongkai Yang. 2017. Unfolding online learning behavioral patterns and their temporal changes of college students in SPOCs. Interactive Learning Environments 25, 2 (2017), 176188.

[2] Damian S Damianov, Lori Kupczynski, Pablo Calafiore, Ekaterina P Damianova, Gökçe Soydemir, and Edgar Gonzalez. 2009. Time spent online and student performance in online business courses: A multinomial logit analysis. Fournal of Economics and Finance Education 8, 2 (2009), 11-22.

[3] Greg C Elvers, Donald J Polzella, and Ken Graetz. 2003. Procrastination in online courses: Performance and attitudinal differences. Teaching of Psychology 30, 2 (2003), 159-162.

[4] Richard M Felder, Linda K Silverman, et al. 1988. Learning and teaching styles in engineering education. Engineering education 78, 7 (1988), 674-681.

[5] Rebecca Ferguson and Doug Clow. 2015. Consistent Commitment: Patterns of Engagement across Time in Massive Open Online Courses (MOOCs). Fournal of Learning Analytics 2, 3 (2015), 55-80.

[6] Kyung Ryung Kim and Eun Hee Seo. 2015. The relationship between procrastination and academic performance: A meta-analysis. Personality and Individual Differences 82 (2015), 26-33.

[7] John S Kinnebrew, James R Segedy, and Gautam Biswas. 2014. Analyzing the temporal evolution of studentsâĂ $Z$ behaviors in open-ended learning environments. Metacognition and learning 9, 2 (2014), 187-215.

[8] Alice Y Kolb and David A Kolb. 2005. Learning styles and learning spaces: Enhancing experiential learning in higher education. Academy of management learning \& education 4, 2 (2005), 193-212.

[9] Vitomir Kovanović, Srećko Joksimović, Dragan Gašević, James Owers, AnneMarie Scott, and Amy Woodgate. 2016. Profiling MOOC course returners: how does student behavior change between two course enrollments? In Proceedings of the Third (2016) ACM Conference on Learning@ Scale. ACM, New York, NY, USA, 269-272.

[10] Chris Masui, Jan Broeckmans, Sarah Doumen, Anne Groenen, and Geert Molenberghs. 2014. Do diligent students perform better? Complex relations between student and course characteristics, study time, and academic performance in higher education. Studies in Higher Education 39, 4 (2014), 621-643.
[11] James W Michaels and Terance D Miethe. 1989. Academic effort and college grades. Social Forces 68, 1 (1989), 309-319.

[12] Nicolas Michinov, Sophie Brunot, Olivier Le Bohec, Jacques Juhel, and Marine Delaval. 2011. Procrastination, participation, and performance in online learning environments. Computers \& Education 56, 1 (2011), 243-252.

[13] Shirin Mojarad, Alfred Essa, Shahin Mojarad, and Ryan S Baker. 2018. Data-driven learner profiling based on clustering student behaviors: learning consistency, pace and effort. In International Conference on Intelligent Tutoring Systems. Springer, New York, NY, USA, 130-139.

[14] Jihyun Park, Kameryn Denaro, Fernando Rodriguez, Padhraic Smyth, and Mark Warschauer. 2017. Detecting changes in student behavior from clickstream data. In Proceedings of the Seventh International Learning Analytics \& Knowledge Conference. ACM, New York, NY, USA, 21-30.

[15] Hilde Patron and Salvador Lopez. 2011. Student effort, consistency, and online performance. Fournal of Educators Online 8, 2 (2011), n2.

[16] E Ashby Plant, K Anders Ericsson, Len Hill, and Kia Asberg. 2005. Why study time does not predict grade point average across college students: Implications of deliberate practice for academic performance. Contemporary Educational Psychology 30, 1 (2005), 96-116.

[17] David Romer. 1993. Do students go to class? Should they? fournal of Economic Perspectives 7, 3 (1993), 167-174.

[18] Howard Schuman, Edward Walsh, Camille Olson, and Barbara Etheridge. 1985. Effort and reward: The assumption that college grades are affected by quantity of study. Social Forces 63, 4 (1985), 945-966.

[19] Daniel T Seaton, Yoav Bergner, Isaac Chuang, Piotr Mitros, and David E Pritchard. 2014. Who does what in a massive open online course? Commun. ACM 57, 4 (2014), 58-65.

[20] Kuang-Cheng Tseng and Shan-Ying Chu. 2010. Traditional versus online courses, efforts, and learning performance. The fournal of Human Resource and Adult Learning 6, 1 (2010), 115.

[21] MM Van den Hurk, HAP Wolfhagen, DHJM Dolmans, and CPM Van der Vleuten. 1998. The relation between time spent on individual study and academic achievement in a problem-based curriculum. Advances in Health Sciences Education 3, 1 (1998), 43-49.

[22] Wei-Kang Wong. 2008. How much time-inconsistency is there and does it matter? Evidence on self-awareness, size, and effects. Fournal of Economic Behavior \& Organization 68, 3-4 (2008), 645-656. 